\title{
Expression of glycine receptors and gephyrin in rat medial vestibular nuclei and flocculi following unilateral labyrinthectomy
}

\author{
WEN ZHOU*, LIU-QING ZHOU*, HONG SHI* , YANG-MING LENG, BO LIU, SU-LIN ZHANG and WEI-JIA KONG \\ Department of Otorhinolaryngology, Union Hospital of Tongji Medical College, \\ Huazhong University of Science and Technology, Wuhan, Hubei 430022, P.R. China
}

Received January 21, 2016; Accepted September 13, 2016

DOI: $10.3892 /$ ijmm.2016.2753

\begin{abstract}
The medial vestibular nucleus (MVN) and the cerebellar flocculus have been known to be the key areas involved in vestibular compensation (VC) following unilateral labyrinthectomy (UL). In this study, we examined the role of gephyrin and glycine receptor (GlyR) in VC using Sprague-Dawley rats, in an aim to gain deeper insight into the mechanisms responsible for VC. The expression of the $\alpha 1$ and $\beta$ subunits of GlyR and gephyrin was immunohistochemically localized in rat MVN and flocculi. The mRNA and protein expression of GlyR ( $\alpha 1$ and $\beta$ subunits) and gephyrin was quantitatively determined by RT-qPCR and western blot analysis at $8 \mathrm{~h}$, and at 1,3 and 7 days following UL. It was found that in the ipsilateral MVN, the mRNA and protein expression of the $\beta$ subunit of GlyR was significantly increased in comparison to the sham-operated $(\mathrm{P}<0.01)$ rats, and in comparison to the contralateral side $(\mathrm{P}<0.01)$ at $8 \mathrm{~h}$ following UL. In the ipsilateral flocculi, GlyR $\beta$ protein expression was significantly elevated $(\mathrm{P}<0.01$ for all), as compared to the sham-operated rats at $8 \mathrm{~h}$, and at 1 and 3 days and to the contralateral side $8 \mathrm{~h}, 1$ and 3 days following UL. No significant differences were observed in the mRNA and protein expression of GlyR $\alpha 1$ and gephyrin in the MVN or flocculi between the two sides (ipsilateral and contralateral) in the UL group, and between the sham-operated group and the UL group at any time point. The findings of our study thus suggest that GlyR plays a major role in the recovery of the resting discharge of the deafferented MVN neurons in the central vestibular system.
\end{abstract}

Correspondence to: Dr Su-Lin Zhang or Dr Wei-Jia Kong, Department of Otorhinolaryngology, Union Hospital of Tongji Medical College, Huazhong University of Science and Technology, 1277 Jiefang Avenue, Wuhan, Hubei 430022, P.R. China

E-mail: zhang_lilac@sina.com

E-mail: entwjkong@hust.edu.cn

*Contributed equally

Abbreviations: CNS, central nervous system; FTNs, flocculus target neurons; GlyRs, glycine receptors; MVN, medial vestibular nucleus; UL, unilateral labyrinthectomy; VC, vestibular compensation

Key words: flocculus, medial vestibular nucleus, gephyrin, glycine receptor, vestibular compensation

\section{Introduction}

Studies have demonstrated that unilateral labyrinthectomy (UL) causes severe oculomotor and postural symptoms in all animal models. The initial symptoms tend to subside within a few days. This rebalancing process is referred to as vestibular compensation (VC) (1-3). VC has been a well-established animal model used for the study of the deafferentation-induced plasticity of the central nervous system (CNS). It is generally believed that $\mathrm{VC}$ is a response to the substantial imbalance between the two sides of the medial vestibular nucleus (MVN), which are linked by the reciprocal commissural inhibitory system. This theory can well explain the drastic asymmetry in the resting discharge of MVN neurons following UL (4). Moreover, a number of studies have suggested that the cerebellum may also be involved in VC (5-9). The flocculus target neurons (FTNs), which are located at the rostral MVN, have been found to partake in vestibular adaptation (10). Additionally, the ipsilateral flocculus is essential for the enhanced intrinsic excitability of MVN neurons following UL $(10,11)$. Therefore, both the cerebellum and MVN may play a role in VC. However, to date, as regards the time course of 'rebalancing' between the bilateral neuronal activities of the vestibular nuclei, the exact cellular and molecular mechanisms remain poorly understood.

Several hypotheses have been proposed regarding the mechanisms of VC. It has been found that glycinergic FTNs surrounded by Purkinje cell terminals project axons to the ipsilateral abducens nucleus $(12,13)$. The response of MVN neurons to vestibular nerve stimulation is modulated by glycinergic, as well as GABAergic inhibitory inputs (14). Bagnall et al demonstrated the existance of commissurally projecting glycinergic neurons in the MVN of mice (15). Li et al (16) reported that the glycine concentration was decreased in the dorsal part of the bilateral lateral vestibular nucleus followng unilateral vestibular ganglionectomy in rats. Vibert et al (17) found that the response of the MVN neurons to glycine in slices was reduced 3 days following labyrinthectomy in guinea pigs. Recently, Lim et al (18) observed a significantly elevated glycinergic quantal current amplitude in mouse contralateral MVN neurons and a higher current frequency in both ipsilateral and contralateral neurons $4 \mathrm{~h}$ post-UL. Therefore, the augmented resting discharge in the deafferented MVN neurons may be ascribed to changes in the number, affinity or effect of the central vestibular glycine receptors (GlyRs). Nonetheless, Eleore et al failed to 
observe any post-UL difference in expression of gephyrin and various GlyR subunits in the bilateral MVNs (19). Thus far, it remains unknown which type of neurons expresses GlyRs and gephyrin in the central vestibular system. In this study, we examined the role of gephyrin and GlyRs in $\mathrm{VC}$, in an aim to gain deeper insight into the mechanisms of VC.

GlyR consists of four $\alpha$ subunits $(\alpha 1-\alpha 4)$ and one $\beta$ subunit. The $\alpha$ subunit is an indispensable subunit capable of forming functional homomeric channels. The $\beta$ subunit orchestrates ligand binding. Consequently, the subunit stoichiometry is $3 \alpha: 2 \beta(20,21)$. The $\alpha 1$ subunit is ubiquitous in the adult brain, while the $\alpha 2$ subunit is highly expressed in the embryonic brain and its expression diminishes with development (22). The $\alpha 3$ and $\alpha 4$ subunits are rare (23). The $\beta$ subunit is expressed throughout the embryonic and adult brain $(24,25)$ and one of its roles is synaptic anchoring of the GlyR through binding to gephyrin $(26,27)$. To better understand the role of gephyrin and GlyR in VC, in this study, we investigated changes in the expression of gephyrin and the $\alpha 1$ and $\beta$ subunits of GlyR in MVN neurons and flocculi at different time points following UL in rats.

\section{Materials and methods}

Animal experiments. A total of 99 male Sprague-Dawley (SD) rats (weighing 200-250 g) was used. Among these, 3 rats were used for immunohistochemistry, 48 for western blot analysis and the other 48 for reverse transcription-quantitative PCR (RT-qPCR). The animals were obtained from the Experimental Animal Center of Tongji Medical College, Huazhong University of Science and Technology (Wuhan, China). All experimental procedures were conducted in strict accordance with 'Guide for the Care and Use of Laboratory Animals' (no. 80-23, NIH publications, revised 1996) formulated by the National Institutes of Health and approved by the Institutional Review Committee on animal research of our college. For western blot analysis and RT-qPCR, the animals were randomly divided into 2 groups as follows: a UL group $(n=24)$ and a sham-operated group $(n=24)$. A total of 6 animals from each group was sacrificed under anesthesia (ketamine-chlorpromazine mixture 10:1, $2 \mathrm{ml} / \mathrm{kg}$, intraperitoneal injection) at $8 \mathrm{~h}$, and at 1, 3 and 7 days following UL or sham operation. The 4 post-operative time points were selected as representative ones of the restoration of VC based on previous studies and animal studies $(1-3,28,29)$.

Immunofluorescence double-labeling. As aforementioned, in 3 rats, the $\alpha 1$ or $\beta$ subunits of GlyR and gephyrin on Purkinje cells (including axon and terminals) in the MVN and flocculi were stained using immunofluorescence double-labeling. The 3 rats were anesthetized deeply with ketamine-chloprromazine mixture (10:1, $2 \mathrm{ml} / \mathrm{kg}$, intraperitoneal injection) and perfused transcardially with $0.9 \%$ physiological saline and then with $4 \%$ paraformaldehyde (in $0.1 \mathrm{M}$ phosphate buffer). After decapitating the bodies, the temporal bones were removed and the brains were dissected. The cerebellum and brain stem portions were fixed in the same fixative for $12 \mathrm{~h}$ at $4^{\circ} \mathrm{C}$ and then rinsed with distilled water for $30 \mathrm{~min}$, dehydrated with a graded alcohol series, cleared in xylene, immersed in paraffin and then embedded in paraffin. Finally, following the rat brain atlas of Paxinos and Watson (30), the cerebellum sections with flocculus and the brainstem sections with MVN were sectioned at $5 \mu \mathrm{m}$ and collected on poly-L-lysine-coated glass slides. The boundaries of the MVN and flocculus were identified based on the brain atlas (30). Purkinje cells (including axons and terminals) in the flocculus and MVN were immunolabeled with, calbindin, an intracellular calcium-binding protein, and a reliable marker for labeleing these cells $(13,31)$. For immunofluorescence labeling, the cerebellum and brain stem samples which were sectioned (into 5- $\mu \mathrm{m}$-thick slices), were formalinfixed, paraffin-embedded, de-waxed, re-hydrated, with antigens retrieved, as previously described (32). The brain sections with flocculi were then incubated with the following primary antibodies: mouse anti-calbindin monoclonal antibody (1:30; ab82812; Abcam, Cambridge, UK) and rabbit polyclonal antibody against the $\alpha 1$ subunit of GlyR (1:100; AB5052; Millipore, Billerica, MA, USA) or the $\beta$ subunit of GlyR (1:50; sc-20134; Santa Cruz Biotechnology, Inc., Paso Robles, CA, USA) or gephyrin (1:100; ab25784; Abcam). Secondary antibodies included Alexa 488 (green) conjugated to donkey anti-mouse IgG $(1: 300 ; 715-545-150)$ and Alexa 594 (red) conjugated to donkey anti-rabbit IgG (1:300; 711-585-152) (both from Jackson Immunoresearch Laboratories Inc., West Grove, PA, USA). In the end, the sections were counterstained with a DAPI mix (Beyotime Institute of Biotechnology, Haimen, China). Subsequently, the sections were observed under a laser scanning confocal microscope (Nikon, Tokyo, Japan) using laser beams of 490 and $590 \mathrm{~nm}$ with appropriate emission filters, $520-530 \mathrm{~nm}$ and $600-630 \mathrm{~nm}$, respectively. Control tests were incubated with PBS instead of the primary antibody and no immunostaining was observed. The size and brightness of the images were adjusted using Adobe Photoshop 5.0 software (Adobe Systems, Inc., San Jose, CA, USA) during the preparation of the figures.

$U L$. With the UL group, the labyrinthectomy was performed on the right side by following the technique previously reported by Zhou et al (32) and Kitahara et al (33). Briefly, the right tympanic bulla was carefully opened via a retroauricular surgical approach. The stapedial artery was cauterized at two points. The contents of the canal ampullae, the utricle and the saccule were aspirated and the wound was sutured. For the sham-operation group, the tympanic bulla was cut open, with the tympanic membrane and ossicles left intact.

$R T$ - $q P C R$. At $8 \mathrm{~h}$, and 1,3 and 7 days following $\mathrm{UL}, 48$ rats $(\mathrm{n}=6$ per group) were sacrificed under anesthesia (ketaminechlorpromazine mixture 10:1,2 ml/kg, intraperitoneal injection). Following decapitation, the bilateral flocculi were carefully removed from the skull. The brains were immediately placed into $0.9 \%$ ice-cold saline for $1 \mathrm{~min}$. According to the rat brain atlas of Paxinos and Watson (30), horizontal brainstem slices including the MVN were taken in order to dissect each nucleus. To take a slice including the MVN, a brainstem slice $(1.5 \mathrm{~mm}$ thickness) just caudal from the abducens nucleus was dissected, and the bilateral MVN were dissected separately under microscopic guidance using a sharp blade, as previously described by Horii et al (34). All these procedures were performed on a chilled plate in order to prevent possible RNA degradation. Total RNA was extracted from the brain tissues using an RNeasy mini kit (Axygen, Hangzhou, China). cDNA was reverse transcribed using a PrimeScript ${ }^{\mathrm{TM}} \mathrm{RT}$ reagent kit with 

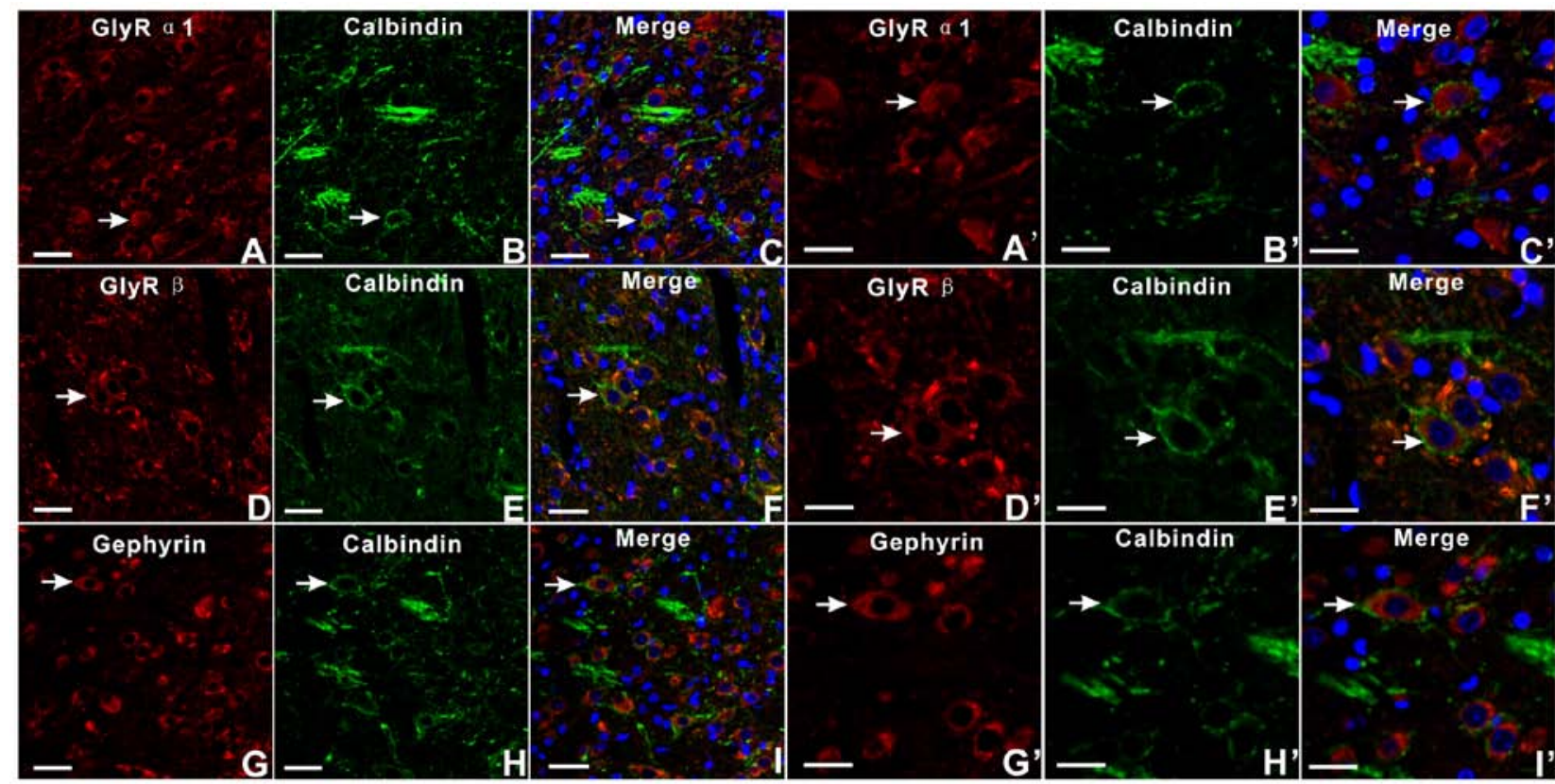

Figure 1. Immunohistochemical findings of glycine receptor (GlyR) $\alpha 1$, GlyR $\beta$ and gephyrin in the medial vestibular nucleus (MVN) under a confocal microscope. Panels A-I and A'-I' show the colocalization of GlyR $\alpha 1$, GlyR $\beta$, gephyrin (red) and calbindin (green) in the MVN. The same cell can be identified by the arrows. Panels A'-C', D'-F' and G'-I' are the enlarged images of cells shown in panels A-C, D-F and G-I, respectively. Scale bar, $50 \mu \mathrm{m}$ in (A-I) and $20 \mu \mathrm{m}$ in (A'-I').

gDNA Eraser (Takara Biotechnology Co., Ltd., Dalian, China). Quantitative (real-time) PCR (qPCR) was performed on a realtime PCR system (model 7300; Applied Biosystems, Foster City, CA, USA). The primer pairs for GlyR $\alpha 1$, GlyR $\beta$, gephyrin and an internal control ( $\beta$-actin) were as follows: GlyR $\alpha 1$ forward, 5'-GTGCTCACCATGACCACACAGA-3' and reverse, 5'-GACACAAAGTTGACAGCGGCATA-3'; GlyR $\beta$ forward, 5'-TGTATGCACCAACCCTGCTGA-3' and reverse, 5'-AGCCACACATCCAGTGCCTTC-3'; gephyrin forward, 5'-CTGGACCCTCGCCCAGAATA-3' and reverse, 5'-CTGTCTTTGGAGGTAGCATCAGCA-3'; $\beta$-actin forward, 5'-CCTGGAGAAGAGCTATGAGC-3' and reverse, 5'-ACAGGATTCCATACCCAGG-3'. The thermal cycling conditions were as follows: $1 \mathrm{~min}$ at $95^{\circ} \mathrm{C}$, and then 40 cycles of $15 \mathrm{sec}$ at $95^{\circ} \mathrm{C}, 20 \mathrm{sec}$ at $60^{\circ} \mathrm{C}$, and $35 \mathrm{sec}$ at $72^{\circ} \mathrm{C}$. Dissociation curves were plotted after each run to ensure primer specificity. The differences $(\Delta \mathrm{Ct})$ in the $\mathrm{Ct}$ values between the target mRNA and the internal control ( $\beta$-actin) were calculated. The changes in the relative mRNA levels were analyzed using the $2^{-\Delta \Delta \mathrm{Ct}}$ method, as described in previous studies $(32,35,36)$.

Western blot analysis. Western blot analysis was performed as previously described $(32,36)$. Briefly, protein from the MVN and flocculi was extracted according to the stereotaxic rat brain atlas of Paxinos and Watson (30). Total protein concentrations were determined using a BCA protein assay kit (Pierce Biotechnology, Inc., Rockford, IL, USA). Ten micrograms of each sample were resolved on an 8\% SDS-PAGE gel. The separated proteins in the trimmed SDS gel were transferred onto PVDF membranes using a transblotting apparatus (Bio-Rad Laboratories, Inc., Hercules, CA, USA), as previously described $(32,36,37,38)$. GlyR $\alpha 1$ (48 kDa) and GlyR $\beta$ (58 kDa) were transferred for $1 \mathrm{~h}$; gephyrin $(93 \mathrm{kDa})$ was transferred for $1.5 \mathrm{~h}$. Prestained protein markers (10-170 kDa; Bio-Rad Laboratories, Inc.) were always run on the same gel. The blots were blocked in 5\% fat-free milk in TBS containing Tween- 20 for $1 \mathrm{~h}$. The membranes were incubated at $4^{\circ} \mathrm{C}$ overnight with affinity-purified polyclonal antibodies against the GlyR $\alpha 1$ (AB5052) and gephyrin (AB5725) (Millipore) diluted at 1:1,000; with monoclonal antibody against the GlyR $\beta$ (sc-365819 Santa Cruz Biotechnology, Inc.) diluted at 1:400. The samples were then incubated with secondary anti-rabbit (111-035-003) or anti-mouse (115-035-003) antibodies (diluted at 1:3,000; both from Jackson Immunoresearch Laboratories Inc.) for $1 \mathrm{~h}$. The monoclonal antibody against GAPDH (GTX627408, GeneTex, Irvine, CA, USA) served as an internal control. The optical density of the protein bands was measured by utilizing the Bio-Rad Quantity One software package (Bio-Rad Laboratories, Inc.), as previously described $(32,36,39)$.

Statistical analysis. Data are expressed as the means \pm SEM and were statistically analyzed using SPSS 13.0 software (SPSS, Inc., Chicago, IL, USA). Statistical significance was evaluated by employing the single analysis of variance (ANOVA), or the Student-Newman-Keuls multiple comparison test or the paired Student's t-test. Statistical significance was set at $\mathrm{P}<0.05$.

\section{Results}

Immunohistochemical findings. Strong immunoreactivity for the $\alpha 1$ and $\beta$ subunits of GlyR and gephyrin was found in all the MVN neurons. The neurons labeled with the $\alpha 1$ and $\beta$ subunits of GlyR and gephyrin were densely surrounded by axonal terminals of Purkinje cells in the MVN (Fig. 1A-I). The same cell can be identified by the arrows. The images shown in panels A'-C', D'-F' and G'-I' are the enlarged images of the cells shown in panels A-C, D-F and G-I, respectively. 


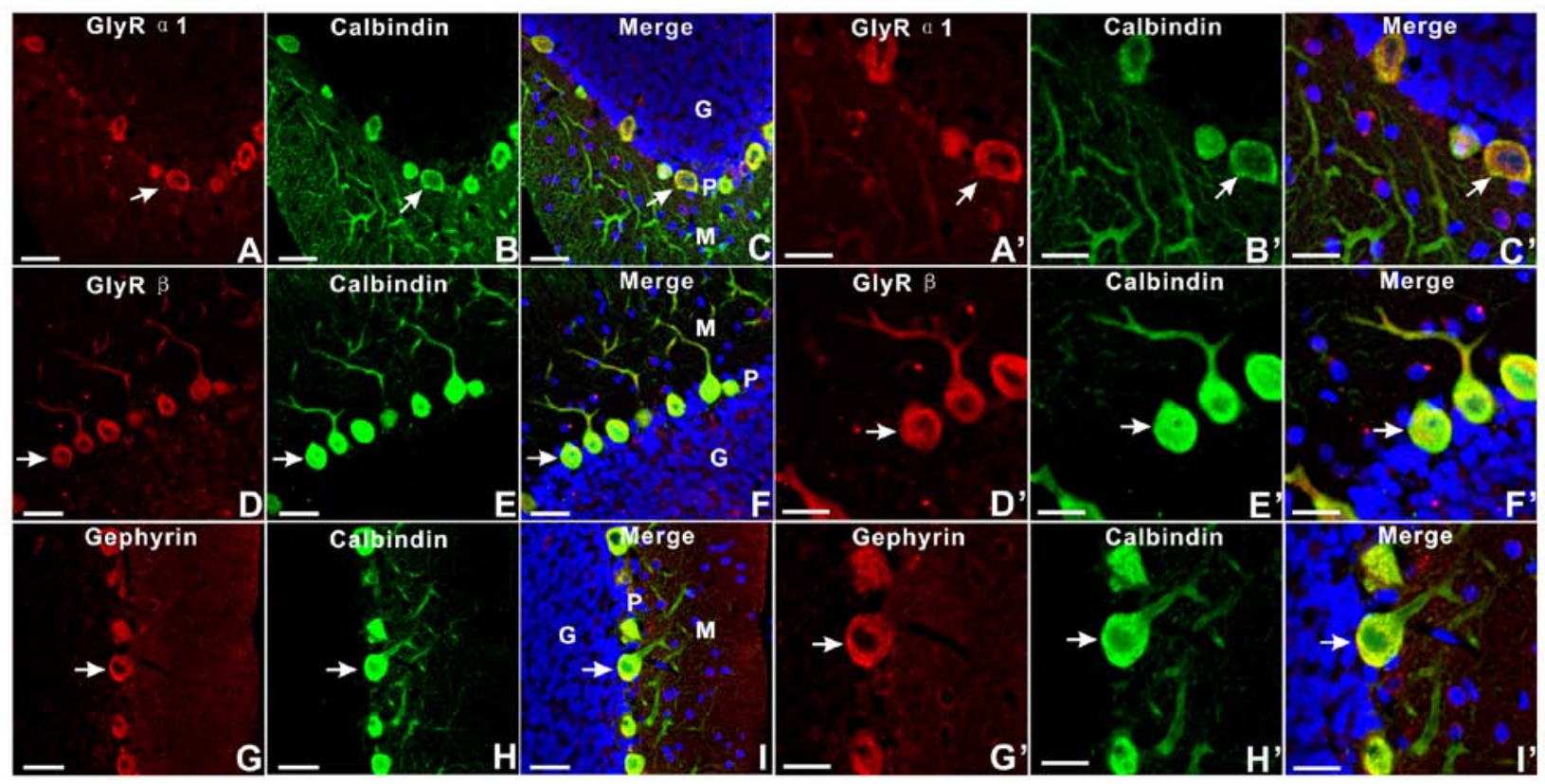

Figure 2. Immunohistochemical findings of glycine receptor (GlyR) $\alpha 1$, GlyR $\beta$ and gephyrin in the flocculi under a confocal microscope. Panels A-I and A'-I' show the colocalization of GlyR $\alpha 1$, GlyR $\beta$, gephyrin (red) and calbindin (green) in the flocculi. G, granular layer; P, Purkinje cell layer; M, molecular layer. The same cell can be identified by the arrows. Panels A'-C', D'-F' and G'-I' are the enlarged images of cells in panels A-C, D-F and G-I, respectively. Scale bar, $50 \mu \mathrm{m}$ in $(\mathrm{A}-\mathrm{I})$ and $20 \mu \mathrm{m}$ in $\left(\mathrm{A}^{\prime}-\mathrm{I}^{\prime}\right)$.
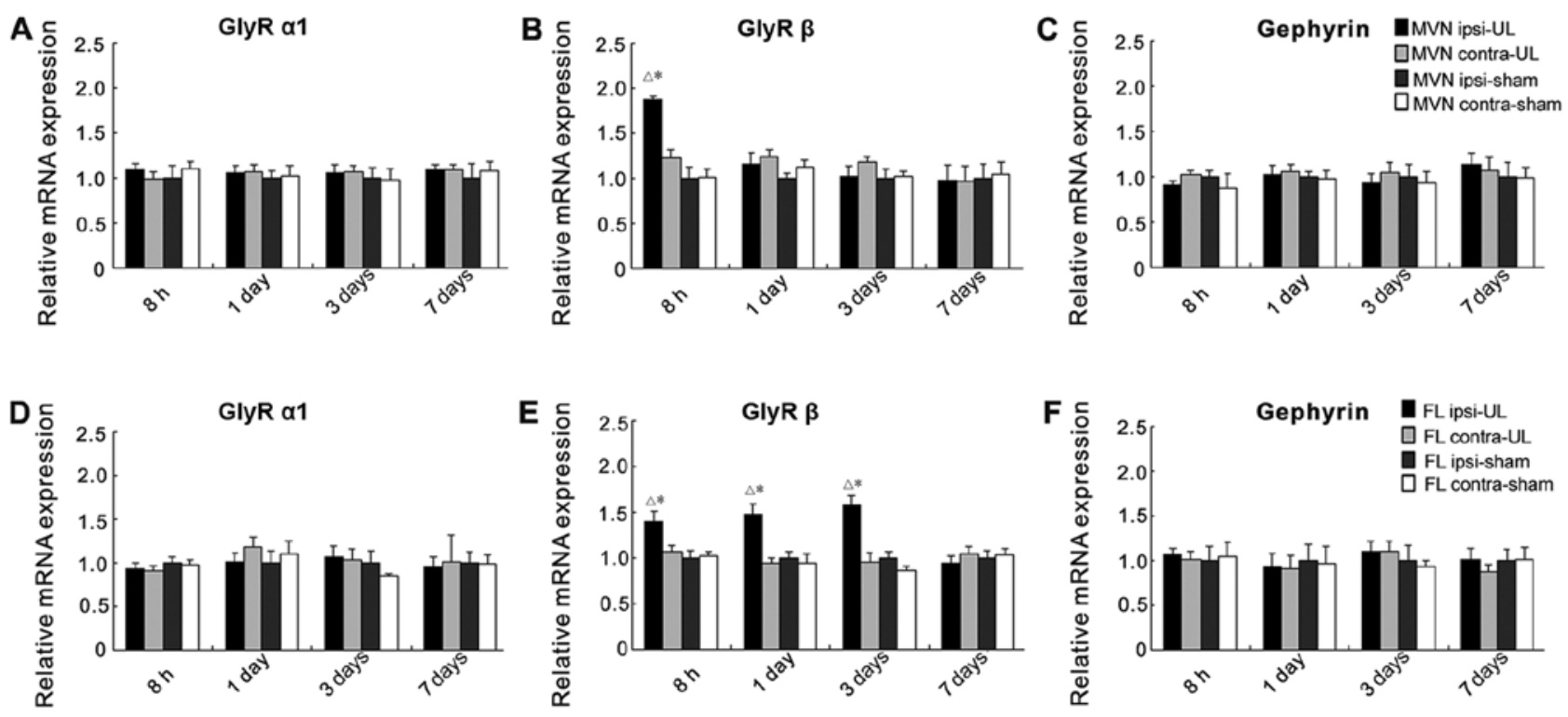

Figure 3. Quantitative determination of the mRNA expression of glycine receptor (GlyR) $\alpha 1$, GlyR $\beta$ and gephyrin at $8 \mathrm{~h}$, and at 1,3 and 7 days following unilateral labyrinthectomy (UL) and in the sham-operated group in (A-C) the medial vestibular nucleus (MVN) and (D-F) the flocculi. Bars represent the means \pm SEM of 6 rats in each group. Ipsi, ipsilateral side; contra, contralateral side; sham, sham-operated group. MVN: ${ }^{\Delta} \mathrm{P}<0.01$ vs. the contralateral side in the UL group at $8 \mathrm{~h} ;{ }^{*} \mathrm{P}<0.01$ vs. the sham-operated group at $8 \mathrm{~h}$. Flocculi: ${ }^{\wedge} \mathrm{P}<0.05$ vs. the contralateral side in the UL group at $8 \mathrm{~h} ; \mathrm{P}<0.01 \mathrm{vs.}$ the contralateral side in the UL group at 1 day; $\mathrm{P}<0.05$ vs. the contralateral side in the UL group at 3 days; ${ }^{*} \mathrm{P}<0.05$ vs. the sham-operated group at $8 \mathrm{~h} ; \mathrm{P}<0.01 \mathrm{vs}$. the sham-operated group at 1 day; and $\mathrm{P}<0.05$ vs. the sham-operated group at 3 days.

In the flocculi, the $\alpha 1$ and $\beta$ subunits of GlyR, and gephyrin (Fig. 2A-I) were moderately to intensely stained in the layers of granular cells and Purkinje cells. The same cell can be identified by the arrows. The images shown in panels A'-C', D'-F' and G'-I' are the enlarged images of the cells shown in panels A-C, D-F and G-I, respectively.

$U L$-induced changes in the mRNA expression of GlyRs and gephyrin in MVN and flocculi. At any time point following UL, no significant differences were observed in the mRNA expression of GlyR $\alpha 1$ (Fig. 3A and D) and gephyrin (Fig. 3C and F) in the MVN or flocculi between the two sides (ipsilateral and contralateral) in the UL group, and between the sham-operated and UL group.

However, the mRNA expression of GlyR $\beta$ in the ipsilateral MVN in the UL group was significantly increased as compared to the sham-operated group $(\mathrm{P}<0.01)$ and to the contralateral MVN $(\mathrm{P}<0.01) 8 \mathrm{~h}$ following UL (Fig. 3B). On 

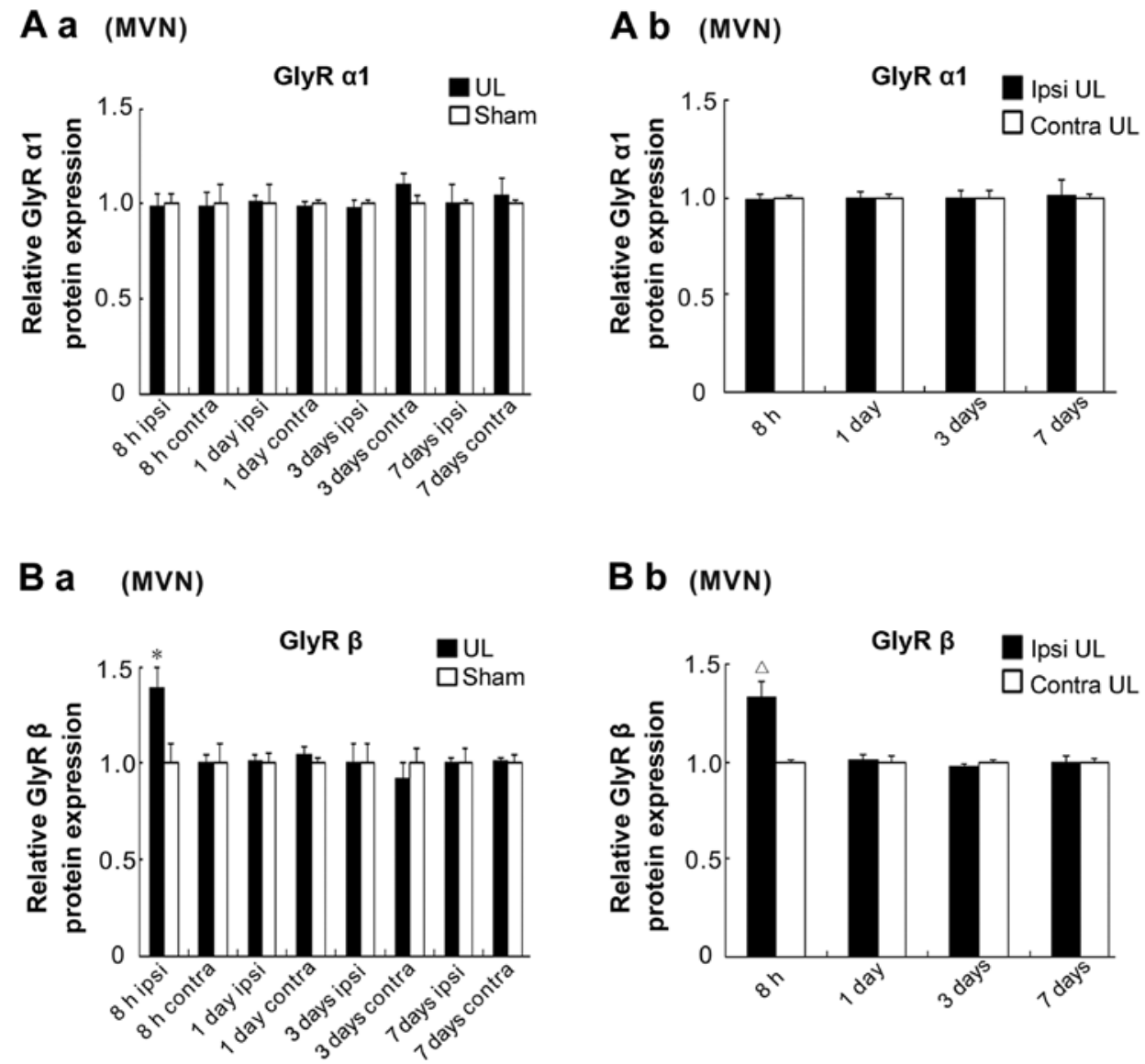

\section{B b (MVN)}
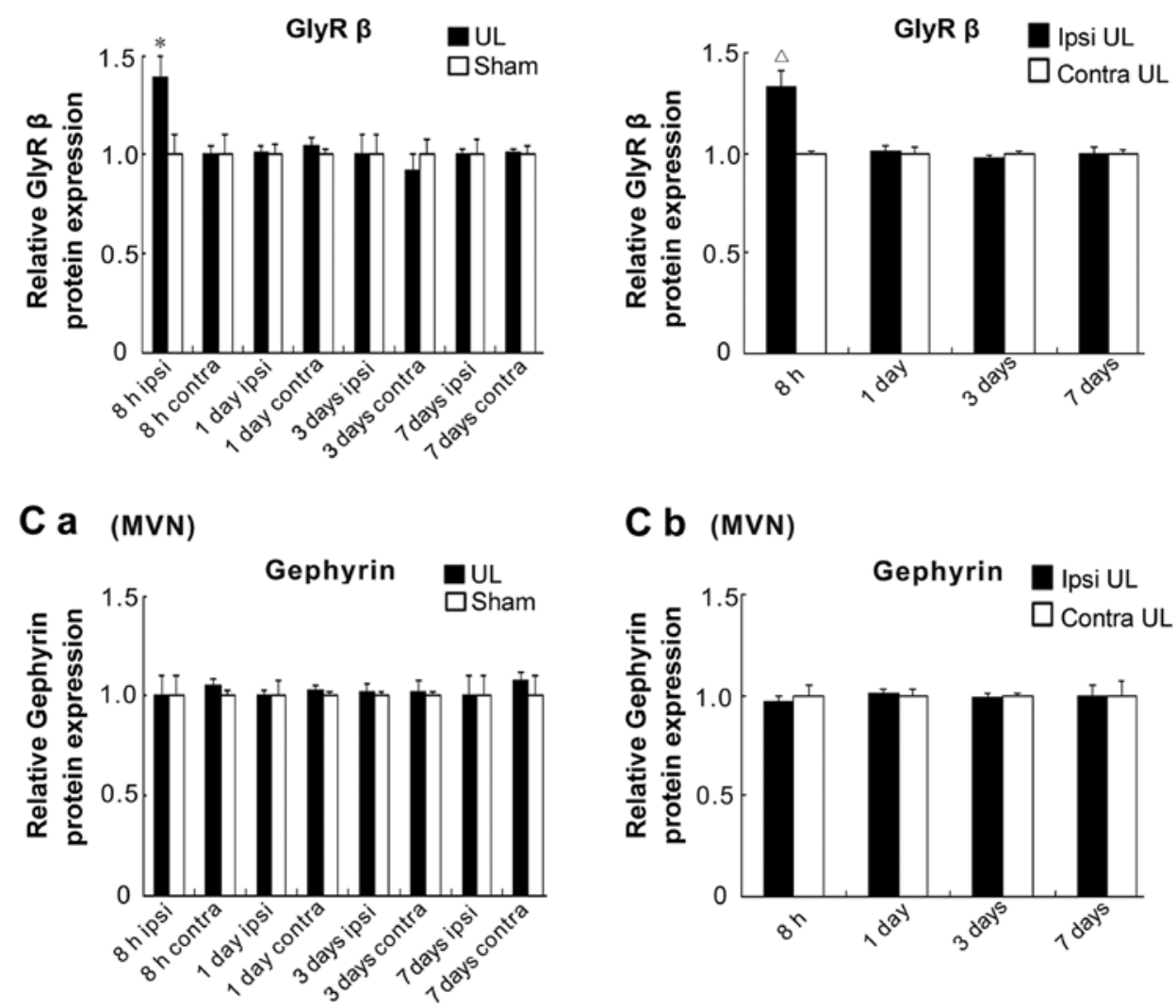

Figure 4. Quantification of glycine receptor (GlyR protein expression in the medial vestibular nucleus (MVN) at $8 \mathrm{~h}$, and at 1,3 and 7 days following unilateral labyrinthectomy (UL). (A, panels a and b) GlyR $\alpha 1$, (B, panels a and b) GlyR $\beta$ and (C, panels a and b) gephyrin protein expression. Ipsi, ipsilateral side; contra, contralateral side; sham, sham-operated group. Bars represent the means \pm SEM of 6 rats in each group. ${ }^{*} \mathrm{P}<0.01$ vs. sham-operated group at 8 h; ${ }^{\Delta} \mathrm{P}<0.01$ vs. contralateral side UL group at $8 \mathrm{~h}$.

the other hand, there were no significant differences in GlyR $\beta$ mRNA expression between the contralateral MVN in the UL group and the sham-operated group (Fig. 3B). Furthermore, in the ipsilateral flocculi, the mRNA expression of GlyR $\beta$ was elevated as compared to the contralateral flocculi and the sham-operated group at $8 \mathrm{~h}(\mathrm{P}<0.05$ vs. contralateral side; $\mathrm{P}<0.05$ vs. sham-operated group), 1 day ( $\mathrm{P}<0.01$ vs. contralateral side; $\mathrm{P}<0.01$ vs. sham-operated group) and 3 days $(\mathrm{P}<0.05$ vs. contralateral side; $\mathrm{P}<0.05$ vs. sham-operated group) following UL (Fig. 3E). By contrast, no significant changes in GlyR $\beta$ mRNA expression were observed between the contralateral flocculi and the sham-operated group (Fig. 3E).
UL-induced changes in the protein levels of GlyRs and gephyrin in MVN and flocculi. In the MVN, the protein expression of both GlyR $\alpha 1$ (Fig. 4A, panels a and b) and gephyrin (Fig. 4C, panels $a$ and $b$ ) exhibited no obvious difference at any time point following UL.

In the flocculi, the protein expression of both GlyR $\alpha 1$ (Fig. 5A, panels a and b) and gephyrin (Fig. 5C, panels a and b) exhibited no obvious difference at any time point following UL.

However, the protein expression of the GlyR $\beta$ in the ipsilateral MVN was significantly increased in comparison to the sham-operated group $(\mathrm{P}<0.01)$ and to the contralateral side $(\mathrm{P}<0.01)$ at $8 \mathrm{~h}$ following UL (Fig. $4 \mathrm{~B}$, panels a and $\mathrm{b})$. On 


\section{A a (flocculus)}

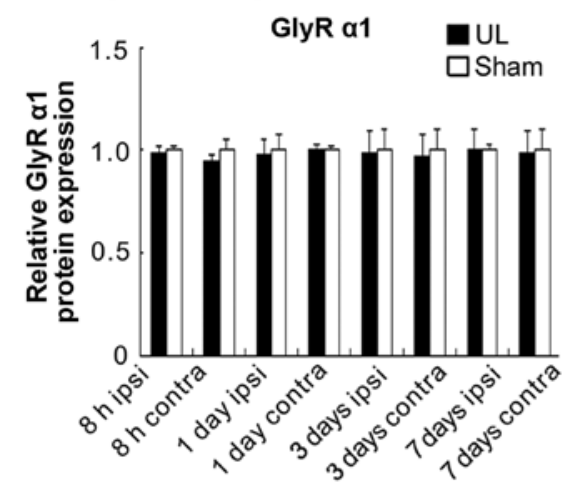

B a (flocculus)

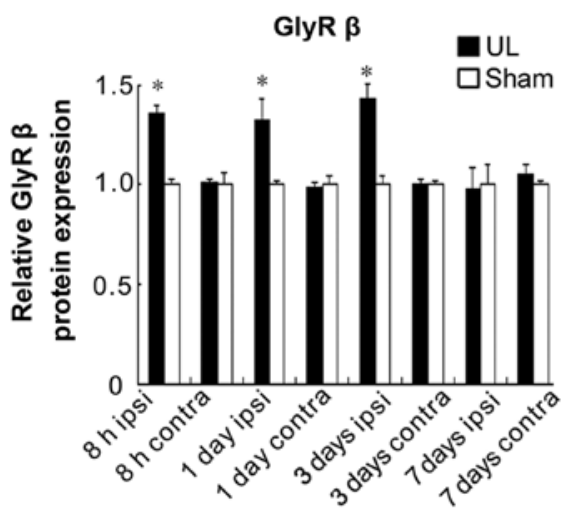

C a (flocculus)

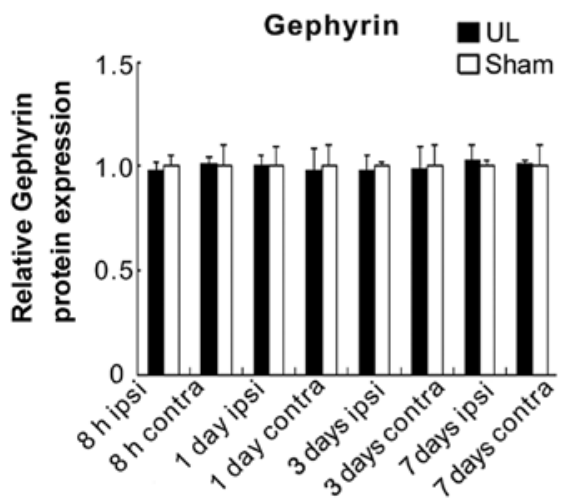

\section{A b (flocculus)}

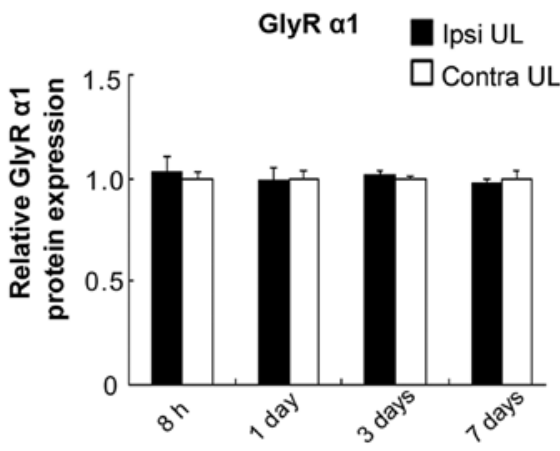

B b (flocculus)

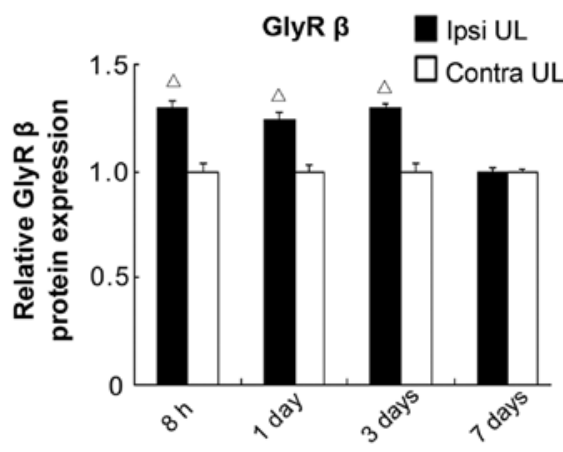

C b (flocculus)

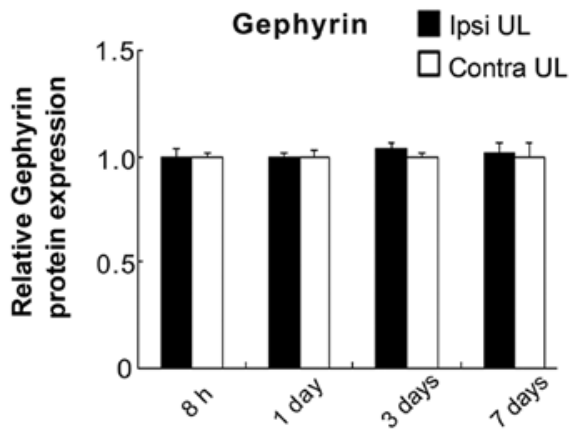

Figure 5. Quantification of glycine receptor (GlyR) protein expression in the flocculus $\approx(\mathrm{FL})$ at $8 \mathrm{~h}, 1,3$ and 7 days following unilateral labyrinthectomy (UL). A , panels a and b) GlyR $\alpha 1$, (B, panels a and b) GlyR $\beta$ and (C, panels a and b) gephyrin protein expression. Ipsi, ipsilateral side; contra, contralateral side; sham, sham-operated group. Bars represent the means \pm SEM of 6 rats/group. ${ }^{*} \mathrm{P}<0.01$ vs. sham-operated group at $8 \mathrm{~h} ; \mathrm{P}<0.01$ vs. sham-operated group at 1 day; $\mathrm{P}<0.01$ vs. sham-operated group at 3 days; ${ }^{\wedge} \mathrm{P}<0.01$ vs. the contralateral side in the UL group at $8 \mathrm{~h} ; \mathrm{P}<0.01$ vs. the contralateral side in the UL group at 1 day; and $\mathrm{P}<0.01$ vs. the contralateral side in the UL group at 3 days.

the other hand, no significant difference was observed in the protein expression of GlyR $\beta$ between the contralateral MVN and the sham-operated group (Fig. 4B, panel a). Furthermore, in the ipsilateral flocculi, GlyR $\beta$ protein expression was significantly increased $(\mathrm{P}<0.01$ for all), as compared to the sham-operated group at $8 \mathrm{~h}$ (Fig. 5B, panel a), 1 day (Fig. 5, panel a) and 3 days (Fig. 5, panel a) following UL, and in comparison to the contralateral side at $8 \mathrm{~h}$ (Fig. 5B, panel b), 1 day (Fig. 5B, panel b) and 3 days (Fig. 5, panel b) following UL. No significant difference in GlyR $\beta$ protein expression was observed between the contralateral flocculi and the shamoperated group (Fig. 5B, panel a).

Fig. 6A shows an immunoblot of GlyR $\beta$ expression in the ipsilateral side in the MVN at $8 \mathrm{~h}$ following UL as compared to the contralateral side in the UL group and to the sham-operated group. Following UL, GlyR $\beta$ expression markedly increased in the MVN in the ipsilaterial side compared to the contralateral side and to the sham-operated group. Fig. 6B-D shows an immunoblot of GlyR $\beta$ in the ipsilateral side in the flocculus at $8 \mathrm{~h}$, and at 1 and 3 days following UL compared to the 


\section{A $(M V N-8 h)$}

U S U S U S U S U S U S

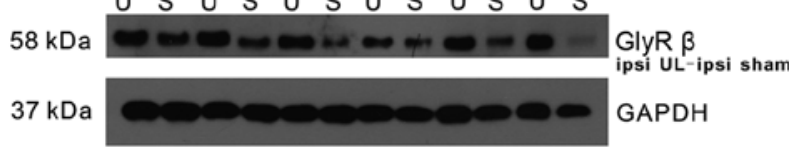

$R \quad L \quad R \quad L \quad R \quad R \quad L \quad R \quad L \quad R \quad L$

$58 \mathrm{kDa}$

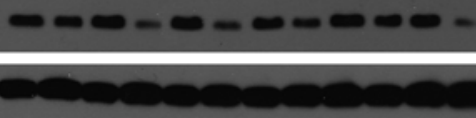

GlyR $\beta$

$37 \mathrm{kDa}$ GAPDH

\section{B (FL-8 h)}

U S U S U S U S S U S

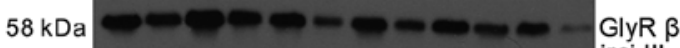

$37 \mathrm{kDa}$ GAPDH

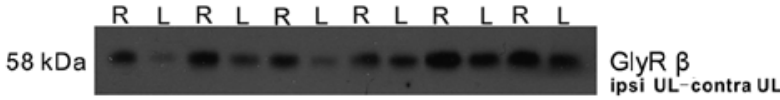

$37 \mathrm{kDa} 0000000000000000000000$
C (FL-1 day)

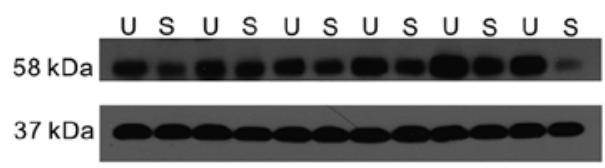

GlyR $\beta$ ipsi UL-ipsi shan GAPDH

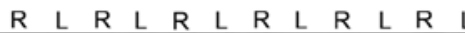

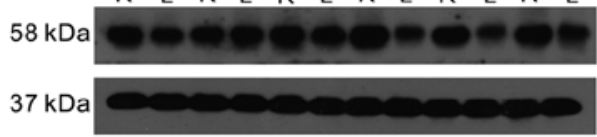

D (FL-3 day)

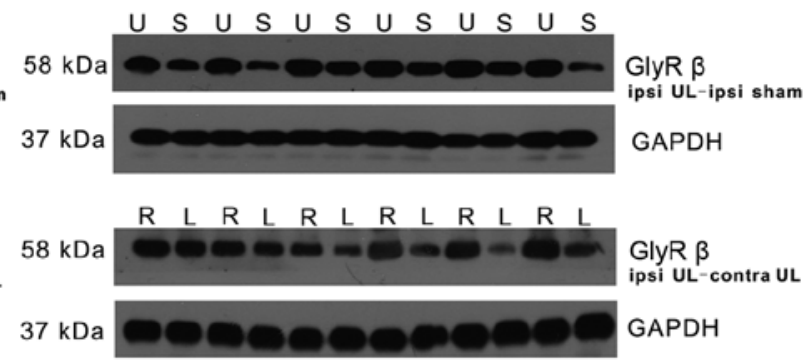

Figure 6. Western blot analysis of glycine receptor (GlyR) $\beta$ in (A) the medial vestibular nucleus (MVN) and (B-D) the flocculus at $8 \mathrm{~h}$, and at 1 and 3 days following unilateral labyrinthectomy (UL). Western blot analysis of GlyR $\beta$ in (A) the MVN and (B-D) in the flocculus compared to the contralateral side and the sham controls at $8 \mathrm{~h}$, and at 1 and 3 days post-UL. (A) Immunoblot of GlyR $\beta$ in the MVN at $8 \mathrm{~h}$ following UL as compared to the contralateral side in the UL and the sham-operated group. (B-D) Immunoblots of GlyR $\beta$ in the flocculus at $8 \mathrm{~h}$, and at 1 and 3 days following UL compared to the contralateral side in the UL and the sham-operated group. U, UL; S, sham; R, ipsi UL; L, contra UL; ipsi, ipsilateral side; contra, contralateral side; sham, sham-operated group; FL, flocculus.
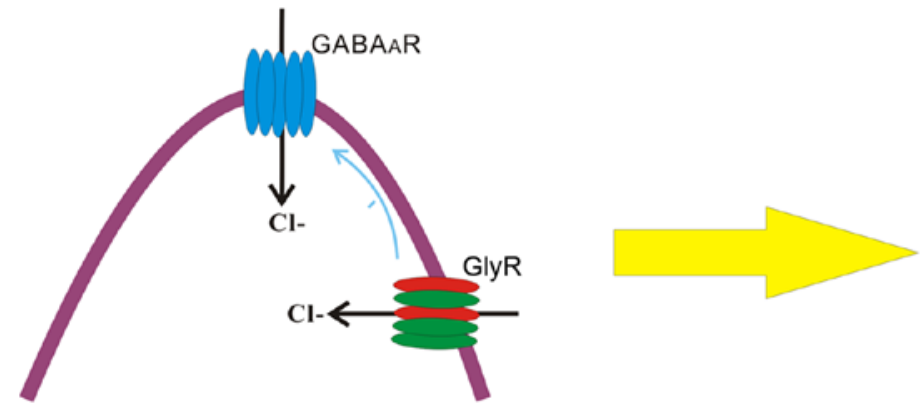
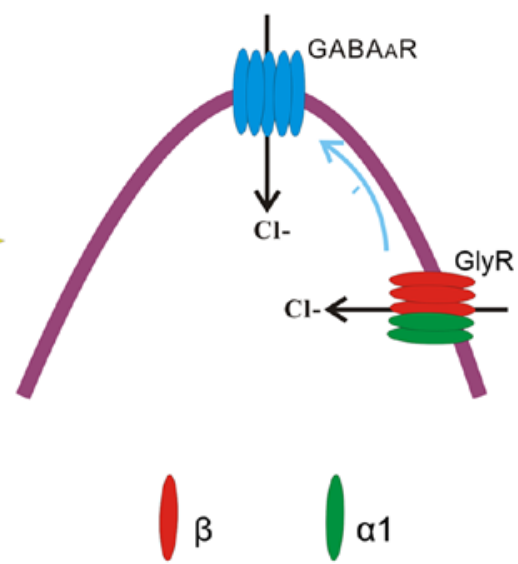

Figure 7. Glycinergic receptor (GlyR) pathways and gephyrin involvement. In heteromeric GlyRs, the $\alpha 1: \beta$ stoichiometry may be $2: 3$ instead of $3: 2$, GlyRs msy modulate excitability by suppressing the GABAergic effect.

contralateral side, and to the sham-operated group. Following UL, GlyR $\beta$ expression was also markedly increased in the ipsilateral side.

\section{Discussion}

This study demonstrated that the GlyR $\beta$ subunit underwent a significant change in expression at the early stage of $\mathrm{VC}$. Following UL, the expression levels of the GlyR $\alpha 1$ subunit and gephyrin in the MVN and flocculi did not exhibit any significant differences at either the mRNA or protein level. These findings suggested that the GlyR $\alpha 1$ subunit or gephyrin may not be involved in VC. Alternatively, the GlyR $\alpha 1$ subunit or gephyrin may experience some functional, not quantitative, changes in the MVN and flocculi during $\mathrm{VC}$ as a result of modification of receptor-binding affinity and its efficacy. However, a previous animal study (19) reported that no asymmetry in the mRNA expression between the two sides of the MVN was detected on autoradiographs at $5 \mathrm{~h}$, and at 1, 3, 8, 30 and 60 days following $\mathrm{UL}$, and in terms of the intensity of immuofluorescence staining at $5 \mathrm{~h}$, and at 1, 3 and 8 days following UL. The discrepancies between our study and the research in question may be that: i) the findings may be differ when different post-UL time points were used; ii) the different experimental procedures used may 
yield different results; and iii) the role of GlyR subunits may vary as a consequence of deafferentation, and the antibody was unable to distinguish between the $\alpha 1$ and $\beta$ subunits of GlyR.

Recently, it has been demonstrated that the suppression of GlyR function decreased the excitability of MVN neurons in mice (40). Inhibitory commissural neurons, which connect the two sides of the MVN, have been shown to be GABAergic and glycinergic $(15,41)$. Several types of neurons in the MVN receive Purkinje cell synapses from the cerebellar flocculus (19). These FTNs involve two types of glycinergic neurons (13). Our immunohistochemical findings were consistent with two previous studies concerning the distribution of GlyR and gephyrin in MVN $(19,42)$. Our study went one step further, in that we found that these GlyR- and gephyrin-expressing neurons were densely surrounded by Purkinje cell terminals. Presumably, GlyR and gephyrin may be present in FTNs. Lu et al (43) observed that post-synaptic GlyRs, when facing GABAergic nerve endings, could be activated by synaptic GABA release in auditory synapses. This raises the possibility that post-synaptic GlyRs, particularly the $\beta$ subunit of GlyR, may be activated by GABA in the FTNs. $\mathrm{Li}$ and $\mathrm{Xu}$ (44) reported that GlyR mediated the downregulation of the $\mathrm{GABA}_{\mathrm{A}}$ receptors. When the GABAergic inhibition is dominant, GlyRs should modulate excitability by suppressing the GABAergic effect (24). In addition, Grudzinska et al (25) suggested that in heteromeric GlyRs, the role of the $\beta$ subunit may be more essential than that of the $\alpha 1$ subunit, therefore the $\alpha 1: \beta$ stoichiometry may be $2: 3$ instead of $3: 2(20,21)$. Therefore, such an increase in the expression of the $\beta$ subunit of GlyR following UL may decrease floccular inhibition on ipsilateral FTNs and contralateral commissural inhibition on ipsilateral MVN neurons (Fig. 7). Thus, a rebalance of the resting activity may be achieved between the ipsilateral and contralateral MVN.

The mRNA and protein expression of the GlyR $\beta$ subunit was significantly increased in the ipsilateral flocculi at $8 \mathrm{~h}$, and at 1 and 3 days following UL. This increase may be ascribed to the different contribution of the $\alpha 1$ and $\beta$ subunits to channel opening (25). Moreover, we also found the $\beta$ subunit of GlyR present in cell bodies and axon terminals of Purkinje cells. Purkinje cells are a major type of inhibitory neurons that release GABA, which acts as a neurotransmitter in the cerebellovestibular pathways (45). Zhang et al (46) reported that GlyRs regulated synaptic plasticity by altering GABAergic neurotransmission. Johnston et al (10) hypothesized that following UL, cerebellar cortical plasticity could induce vestibular neuronal plasticity. On the basis of these findings, we came to the conclusion that, following UL, the upregulated expression of the $\beta$ subunit of GlyR may contribute to cerebellar cortical plasticity. As a result, a rebalance of the neuronal activity was attained between the ipsilateral and contralateral MVN.

In conclusion, GlyR may play a major role in the recovery of the resting discharge of the deafferented MVN neurons in the central vestibular system. However, the mechanisms through which the $\beta$ subunit of GlyR functions in the central vestibular system during $\mathrm{VC}$ warrants further investigation.

\section{Acknowledgements}

This study was supported by grants from the National 12th-Five Year Research Program of China (no. 2012BAI12B02), the Key
Project of the National 11th-Five Year Research Program of China (no. 2007BAI18B13) and the National Natural Science Foundation of China (no. 81500792).

\section{References}

1. Smith PF and Curthoys IS: Mechanisms of recovery following unilateral labyrinthectomy: a review. Brain Res Brain Res Rev 14: 155-180, 1989.

2. Curthoys IS and Halmagyi GM: Vestibular compensation. Adv Otorhinolaryngol 55: 82-110, 1999.

3. Dieringer N: 'Vestibular compensation': neural plasticity and its relations to functional recovery after labyrinthine lesions in frogs and other vertebrates. Prog Neurobiol 46: 97-129, 1995.

4. Bergquist F, Ludwig M and Dutia MB: Role of the commissural inhibitory system in vestibular compensation in the rat. $\mathrm{J}$ Physiol 586: 4441-4452, 2008.

5. Beraneck M, McKee JL, Aleisa M and Cullen KE: Asymmetric recovery in cerebellar-deficient mice following unilateral labyrinthectomy. J Neurophysiol 100: 945-958, 2008.

6. Dutia MB: Mechanisms of vestibular compensation: recent advances. Curr Opin Otolaryngol Head Neck Surg 18: 420-424, 2010.

7. Kitahara T, Takeda N, Kiyama H and Kubo T: Molecular mechanisms of vestibular compensation in the central vestibular system - review. Acta Otolaryngol Suppl 539: 19-27, 1998.

8. Kitahara T, Takeda N, Saika T, Kubo T and Kiyama H: Role of the flocculus in the development of vestibular compensation: immunohistochemical studies with retrograde tracing and flocculectomy using Fos expression as a marker in the rat brainstem. Neuroscience 76: 571-580, 1997.

9. Courjon JH, Flandrin JM, Jeannerod M and Schmid R: The role of the flocculus in vestibular compensation after hemilabyrinthectomy. Brain Res 239: 251-257, 1982.

10. Johnston AR, Seck1 JR and Dutia MB: Role of the flocculus in mediating vestibular nucleus neuron plasticity during vestibular compensation in the rat. J Physiol 545: 903-911, 2002.

11. Nelson AB, Krispel CM, Sekirnjak C and du Lac S: Long-lasting increases in intrinsic excitability triggered by inhibition. Neuron 40: 609-620, 2003.

12. de Waele C, Mühlethaler M and Vidal PP: Neurochemistry of the central vestibular pathways. Brain Res Brain Res Rev 20: 24-46, 1995.

13. Shin M, Moghadam SH, Sekirnjak C, Bagnall MW, Kolkman KE, Jacobs R, Faulstich M and du Lac S: Multiple types of cerebellar target neurons and their circuitry in the vestibulo-ocular reflex. J Neurosci 31: 10776-10786, 2011.

14. Biesdorf S, Malinvaud D, Reichenberger I, Pfanzelt S and Straka H: Differential inhibitory control of semicircular canal nerve afferent-evoked inputs in second-order vestibular neurons by glycinergic and GABAergic circuits. J Neurophysiol 99: 1758-1769, 2008.

15. Bagnall MW, Stevens RJ and du Lac S: Transgenic mouse lines subdivide medial vestibular nucleus neurons into discrete, neurochemically distinct populations. J Neurosci 27: 2318-2330, 2007.

16. Li H, Godfrey TG, Godfrey DA and Rubin AM: Quantitative changes of amino acid distributions in the rat vestibular nuclear complex after unilateral vestibular ganglionectomy. J Neurochem 66: 1550-1564, 1996.

17. Vibert N, Beraneck M, Bantikyan A and Vidal PP: Vestibular compensation modifies the sensitivity of vestibular neurones to inhibitory amino acids. Neuroreport 11: 1921-1927, 2000.

18. Lim R, Callister RJ and Brichta AM: An increase in glycinergic quantal amplitude and frequency during early vestibular compensation in mouse. J Neurophysiol 103: 16-24, 2010.

19. Eleore L, Vassias I, Vidal PP and de Waele C: An in situ hybridization and immunofluorescence study of glycinergic receptors and gephyrin in the vestibular nuclei of the intact and unilaterally labyrinthectomized rat. Exp Brain Res 154: 333-344, 2004.

20. Lynch JW: Molecular structure and function of the glycine receptor chloride channel. Physiol Rev 84: 1051-1095, 2004.

21. Burzomato V, Groot-Kormelink PJ, Sivilotti LG and Beato M: Stoichiometry of recombinant heteromeric glycine receptors revealed by a pore-lining region point mutation. Receptors Channels 9: 353-361, 2003.

22. Sato K, Kiyama $\mathrm{H}$ and Tohyama M: Regional distribution of cells expressing glycine receptor alpha 2 subunit mRNA in the rat brain. Brain Res 590: 95-108, 1992. 
23. Matzenbach B, Maulet Y, Sefton L, Courtier B, Avner P, Guénet JL and Betz H: Structural analysis of mouse glycine receptor alpha subunit genes. Identification and chromosomal localization of a novel variant. J Biol Chem 269: 2607-2612, 1994

24. Xu TL and Gong N: Glycine and glycine receptor signaling in hippocampal neurons: diversity, function and regulation. Prog Neurobiol 91: 349-361, 2010.

25. Grudzinska J, Schemm R, Haeger S, Nicke A, Schmalzing G, Betz $\mathrm{H}$ and Laube B: The beta subunit determines the ligand binding properties of synaptic glycine receptors. Neuron 45: 727-739, 2005.

26. Meyer G, Kirsch J, Betz H and Langosch D: Identification of a gephyrin binding motif on the glycine receptor beta subunit. Neuron 15: 563-572, 1995.

27. Sola M,Bavro VN,Timmins J,FranzT,Ricard-BlumS,Schoehn G Ruigrok RW, Paarmann I, Saiyed T, O'Sullivan GA, et al: Structural basis of dynamic glycine receptor clustering by gephyrin. EMBO J 23: 2510-2519, 2004.

28. Darlington CL and Smith PF: Molecular mechanisms of recovery from vestibular damage in mammals: recent advances. Prog Neurobiol 62: 313-325, 2000.

29. Zhou L, Zhou W and Zhang S, Liu B, Liang P, Zhou Y, Zhou T, Zhang K, Leng $\mathrm{Y}$ and Kong W: BDNF signaling in the rat cerebello-vestibular pathway during vestibular compensation: BDNF signaling in vestibular compensation. FEBS J 282: 3579-3591, 2015

30. Paxinos G and Watson C: The Rat Brain in Stereotaxic Coordinates. Academic Press, New York, pp16-24, 2007.

31. Bäurle J and Grüsser-Cornehls U: Calbindin D-28k in the lateral vestibular nucleus of mutant mice as a tool to reveal Purkinje cell plasticity. Neurosci Lett 167: 85-88, 1994.

32. Zhou W, Zhou LQ, Zhang SL, Liu B, Leng YM, Zhou RH and Kong WJ: The changes in mGluR2 and mGluR7 expression in rat medial vestibular nucleus and flocculus following unilateral labyrinthectomy. Int J Mol Sci 14: 22857-22875, 2013.

33. Kitahara T, Takeda N, Emson PC, Kubo T and Kiyama H: Changes in nitric oxide synthase-like immunoreactivities in unipolar brush cells in the rat cerebellar flocculus after unilateral labyrinthectomy. Brain Res 765: 1-6, 1997.

34. Horii A, Smith PF and Darlington CL: Quantitative changes in gene expression of glutamate receptor subunits/subtypes in the vestibular nucleus, inferior olive and flocculus before and following unilateral labyrinthectomy in the rat: real-time quantitative PCR method. Exp Brain Res 139: 188-200, 2001.
35. Livak KJ and Schmittgen TD: Analysis of relative gene expression data using real-time quantitative PCR and the 2(-Delta Delta C(T)) method. Methods 25: 402-408, 2001.

36. Zhou L, Zhou W, Zhang S, Liu B, Leng Y, Zhou R and Kong W: Changes in histamine receptors ( $\mathrm{H} 1, \mathrm{H} 2$, and $\mathrm{H} 3$ ) expression in rat medial vestibular nucleus and flocculus after unilateral labyrinthectomy: histamine receptors in vestibular compensation. PLoS One 8: e66684, 2013.

37. Zheng Y, Horii A, Appleton I, Darlington CL and Smith PF: Damage to the vestibular inner ear causes long-term changes in neuronal nitric oxide synthase expression in the rat hippocampus. Neuroscience 105: 1-5, 2001.

38. Ashton JC, Little E, Muir M, Smith PF and Darlington CL: Mitochondrial ultrastructure and apoptotic protein expression in the vestibular nucleus complex following unilateral labyrinthectomy. Brain Res 1055: 165-170, 2005.

39. Liu P, Zheng Y, King J, Darlington CL and Smith PF: Long-term changes in hippocampal n-methyl-D-aspartate receptor subunits following unilateral vestibular damage in rat. Neuroscience 117 965-970, 2003.

40. Camp AJ, Lim R, Anderson WB, Schofield PR, Callister RJ and Brichta AM: Attenuated glycine receptor function reduces excitability of mouse medial vestibular nucleus neurons. Neuroscience 170: 348-360, 2010.

41. Holstein GR, Martinelli GP and Cohen B: The ultrastructure of GABA-immunoreactive vestibular commissural neurons related to velocity storage in the monkey. Neuroscience 93: 171-181, 1999.

42. Friauf E, Hammerschmidt B and Kirsch J: Development of adult-type inhibitory glycine receptors in the central auditory system of rats. J Comp Neurol 385: 117-134, 1997.

43. Lu T, Rubio ME and Trussell LO: Glycinergic transmission shaped by the corelease of GABA in a mammalian auditory synapse. Neuron 57: 524-535, 2008

44. Li Y and $\mathrm{Xu}$ TL: State-dependent cross-inhibition between anionic GABA(A) and glycine ionotropic receptors in rat hippocampal CA1 neurons. Neuroreport 13: 223-226, 2002.

45. Obata K and Takeda K: Release of gamma-aminobutyric acid into the fourth ventricle induced by stimulation of the cat's cerebellum. J Neurochem 16: 1043-1047, 1969.

46. Zhang LH, Xu L and Xu TL: Glycine receptor activation regulates short-term plasticity in CA1 area of hippocampal slices of rats. Biochem Biophys Res Commun 344: 721-726, 2006. 